\title{
Hydrodynamic Time Scale of Resonance in Intense Laser Irradiated Nano-Plasmaof Different Geometries
}

\author{
Uday Chakravarty $^{1,2^{*}}$, Deepa Chaturvedi ${ }^{3}$ \\ ${ }^{1}$ Laser Plasma Section, Raja Ramanna Centre for Advanced Technology, Indore, India 452013 \\ ${ }^{2}$ Homi Bhabha National Institute, Training School Complex, Anushaktinagar, Mumbai, India 400094 \\ ${ }^{3}$ Indian Institure of Technolgy, Delhi, India 110016 \\ *Corresponding Author: uday@rrcat.gov.in, Tel.: 8435718289
}

Available online at: www.isroset.org

Received: 22/May/2018, Revised: 06/Jun/2018, Accepted: 27/Jun/2018, Online: 30/Jun/2018

\begin{abstract}
Absorption of intense ultra-short laser pulse $10^{15} \mathrm{~W} / \mathrm{cm}^{2}<<\mathrm{I} \leq 10^{18} \mathrm{~W} / \mathrm{cm}^{2}$ in hot dense plasmais inefficient.At high temperatures plasma becomes literally collision less andabsorptioncould only be achieved by excitation of resonance. For realizing resonance nanostructures are used and for high absorption laser parameters like intensity and pulse duration should be judiciously chosen according to the shape of nanostructure. Intense laser heated hydro-dynamically expanding nanostructures undergo electron density dilution and their natural frequency of charge density oscillation meet the laser frequency for excitation of resonance.It is found that solid nano-particles like gas clusters and nano-rods, the hydrodynamic time scales to reach resonance is longer in comparison to hollow nanostructures like nano-tubes and nano-shells. It is also shown that due to polarization pressure solid nanostructure undergo expansion primarily along the incident laser polarization; this also leads to arrival of resonance condition earlier in time. It is desirable that the resonance occurs at high density for a significant radiation and particle flux. For this hollow nanostructures or higher laser intensity pulse is required for resonance condition to occur at higher density even for ultrashort pulses. This analytical analysis gives a handy recipe for choosing the laser Fluence/Intensity and pulse duration so that the resonance condition is met at the peak of the incident laser pulse. Also, the derived expressions for hydrodynamic time scales of resonance serve as a guide for choosing laser and target geometrical parameters for efficient laser energy coupling in nano-plasmas for efficient X-rays, electron and ion generation.
\end{abstract}

Keywords-Resonance, Nano-structures, Gas Clusters, Nano-rod, Nano-shell, Nano-tube, Elliptical Nano-cylinders, Hydrodynmaics of plasma, Ultrashort Lasers, Collisionless plasmas

\section{INTRODUCTION}

Absorption of intense laser pulses in plasmas is a widely pursued challenging problem [1]. Coupling of laser energy in hot dense matter is paramount to efficient X-rays [2], Hot Ions [3], and Fast electron generation [4]. They have a widespread application in inertial confinement fusion [5], Radiation and particle radiography [6], Mammography [7], Cancer cure [8]. Various technological endeavours with futuristic progress and development promise, are undertaken from the interesting discoveries in the field of laser plasma interaction. However, absorption of intense ultra-short pulse in matter is a long standing problem [1,9]. Since at high intensities the temperature of the laser heated matter becomes $>1 \mathrm{keV}$ the plasma becomes collisionless $[9,10]$. It is well known from the Poynting's theorem that absorption of light in matter should be accompanied by dephasing between the incident laser field $\mathrm{E}$ and resultant current density $\mathrm{J}$ [11]. Collisions cause a natural dephasing and hence are responsible for energy deposition in matter. However, in absence of collisions a scenario that is encountered in intense laser matter interaction dephasing can be caused if resonance is excited [12]. It is also known that at resonance there is a polarizability reversal and occurrence of drastic phase change, therefore resonance is the key to laser energy coupling in matter in absence of collisions. Therefore it is not a surprise that a wide number of studies have been devoted to finding targets like nanostructures of various shape, size, morphology and geometry for efficient laser energy coupling [13].For this purpose solid nanostructures like spherical gas clusters [14], metal clusters [15], nano-rods [16], nano-tubes [17], nano-shells etc. are used. The resonance supported by them depends on their shape and geometry [18]. Only little study has been done previously to analytically predict the time scales / laser pulse duration and intensity required for exciting resonance in nanostructures of different shapes and geometries. In this work we analytically predict the hydrodynamic time scales for occurrence of resonance in various nanostructures of different geometries. In addition to this the role of laser intensity in accelerating the density 
dilution process during the hydrodynamic expansion is investigated. The paper is organised as follows. First in section II we give a basic introduction to light nano-structure interaction. In section III physics of resonance is discussed. Here we derive the resonance condition of solid spherical and nano-rods. This is followed by a brief overview on hollow nano-structures like nano-tubes and nano-shells in section IV. Interestingly hollow nanostructures exhibit two resonances and therefore have two instances when resonance condition is met during the hydrodynamic expansion. In section $\mathrm{V}$ the electric field and resonance density in elliptical nano-rod is shown. Section VI is devoted to finding the hydrodynamic time scales of resonance in solid, hollow and pointed nano-structures. The effect of polarisation pressure in making the expansion predominantly along the laser polarisation direction is elaborated in section VI. These results in the nano-rod shape modification to become nanoelliptical causing an increase of its resonance density. The derived expressions are used to produce various graphs in section VII. These graphs convey very easily the time scales of resonance in various nano-particles of different size and interacting at different laser intensities. It is shown that at high intensity the laser intensity is quite crucial for determining the time scales of occurrence of resonance. Resonance occurring at high density is important, and for this hollow and pointed nanostructures should be used. The need for exciting resonance at the peak of laser pulse demands that the pulse duration should be stretched, leading to lowering of intensity [10]. This can be avoided by using higher intensities to accelerate the expansion rate of nanoparticles and resonance can occur at the peak of pulse with considerably low pulse stretching. Section VIII concludes the work and gives future perspective.

\section{LIGHT NANO-STRUCTURE INTERACTION}

First we outline the basic assumptions used to determine the hydrodynamic time scales of resonance in laser irradiated nano-structures. The incident laser light is linearly polarised and intensity $\gg 10^{15} \mathrm{~W} / \mathrm{cm}^{2}$, pulse duration may be $100 \mathrm{fs}-1$ ps (ultra-short laser pulses). The incident laser wavelength is $800 \mathrm{~nm}$ Ti: Sa Laser and $\lambda \gg$ size of nano-particle. Under this assumption quasi-static approximation is used treating light matter interaction as dielectric kept in electric field [19]. For cylindrical nano-particles $\lambda>>$ radius of the nanocylinder [20]. The resonance density of different nanostructure is derived by solving the Laplace equation in proper coordinate system according to the nanostructure geometry. The normal component of electric displacement vector and tangential component of the electric field is continuous across the boundary. For hollow structures these boundary conditions are applied for both inner and outer surface [20]. Also the electric potential is continuous at the boundary. Another assumption which is also validated in other works is that laser ionizes the nano-structure at the foot of the pulse through tunnel and field ionisation and a solid density nano-plasma is created $[18,19]$. The heated nanostructure expands due to a combination of Hydrodynamic, Coulomb [19] and Polarisation pressure. Hydrodynamic pressure is predominant for large diameter nano-particle and polarisation pressure is dominant for pointed nano-structures. The energy decrease/cooling due to expansion is compensated by the incoming supply of laser pulse energy. Therefore the average temperature of the cluster during the hydrodynamic expansion does not strongly depend on laser pulse duration. But temperature is sensitive to the Fluence /Intensity of the pulse (Where intensity is varied by changing the laser energy).Moreover, ionisation produces more electrons as the laser instantaneous intensity increases, but the energy redistribution keeps the temperature almost constant. So the average temperature during the entire evolution dynamics determines the hydrodynamic expansion rate and is considered to depend only on laser Fluence or Intensity [21].The polarization of the nano-particle dynamically varies with laser frequency and depends on the instantaneous electron density (n) and the geometry of the structure. Sufficient hydrodynamic expansion is needed for density dilution of the solid density nano-plasma $\left(\mathrm{n}_{0}\right)$; so that its natural instantaneous oscillation frequency becomes equal to the laser frequency $\omega$ (Resonance condition). This happens when the diluted instantaneous electron density (n) becomes equal to the resonance density [22].

\section{RESONANCE IN SOlid NANO-STRUCTURES}

The electric field inside a dielectric sphere $\left(E_{\text {in }}\right)$ is well known and the resonance condition can be derived from it. At resonance polarisation reversal take place and electric field blows to a high magnitude. It may be noted that the field enhancement at resonance is instrumental in the efficient particle and radiation generation since the effective intensity of light also escalates to a very high value.

$$
E_{\text {in }}=\frac{3 E_{0}}{\varepsilon+2}
$$

Here $\mathrm{E}_{0}$ is the applied laser field. The dielectric constant $\varepsilon$ of plasma is given by

$$
\left(\varepsilon=1-\frac{\mathrm{n}_{\mathrm{e}} / \mathrm{n}_{\mathrm{c}}}{(1+\mathrm{i} v / \omega)}\right)
$$

It depends on electron density $n_{e}$, critical density $n_{c}$ (which is $1.7 \mathrm{X} 10^{21} /$ c.c. for $800 \mathrm{~nm}$ light ) and the collision frequency $v$. The Spitzer collision frequency is given by eq.(3) 


$$
v_{e, i} \approx 3 \times 10^{-6} \ln \Lambda n_{e}\left(\frac{Z}{T_{e}^{3 / 2}}\right)
$$

Where $\ln \Lambda$ is the Coulomb logarithm, $T_{e}$ is the electron temperature and $\mathrm{Z}$ is the degree of ionization. At high intensity the collision frequency gets modified due to effective temperature of the electron moving in electric field. The modified value of temperature $\mathrm{T}_{\text {eff }}$ is put in eq.(3)using eq. (4). $\mathrm{T}_{\text {eff }}(\mathrm{eV})$ depends on $\mathrm{U}_{\mathrm{p}}=9.33 \mathrm{I}_{14}\left(\mathrm{~W} / \mathrm{cm}^{2}\right) \lambda(\mu \mathrm{m})^{2}$ which is the average oscillatory energy of electron in laser field. At high intensity $\mathrm{I}>>10^{15} \mathrm{~W} / \mathrm{cm}^{2} \mathrm{~T}_{\text {eff }}$ becomes $>1 \mathrm{keV}$ and collision frequency becomes very low implying plasma becomes collisonless.

$$
T_{e f f}=\sqrt{T_{e}^{2}+\left(U_{p} / k_{B}\right)^{2}}
$$

For a collisionless plasma resonance density just depends on electron density. From eq.(1) resonance density is obtained by putting the denominator $(\varepsilon+2)=0$.The inferences drawn using eq. (2), (3) and (4) yield the resonance density of spherical nano-particle as $[19,21]$

$$
n_{e}=3 n_{c}
$$

Similarly for plasma dielectric in the shape of solid nano-rod placed in the laser field, where the electric field is perpendicular to cylinder axis is given by [23]

$$
E_{\text {in }}=\frac{2 E_{0}}{\varepsilon+1}
$$

And the resonance condition in solid nano-rod is

$$
n_{e}=2 n_{c}
$$

\section{RESONANCE IN HOLLOW NANO-STRUCTURES}

The radial and angular averaged electric field inside a nanotube (inner radius ' $a$ ' and outer radius ' $b$ ') like "carbon nanotubes" (CNT) is given by ( $\mathrm{E}_{0}$ is perpendicular to the axis) [20]

$$
\begin{gathered}
\left\langle E_{\text {in }}\right\rangle=\sqrt{A_{2}^{2}+\frac{B_{2}^{2}}{a^{2} b^{2}}} \\
A_{2}=\frac{-2 E_{0}(\varepsilon+1)}{(\varepsilon+1)^{2}-\left(\frac{a}{b}\right)^{2}(\varepsilon-1)^{2}} ; B_{2}=\frac{-2 E_{0} a^{2}(\varepsilon-1)}{(\varepsilon+1)^{2}-\left(\frac{a}{b}\right)^{2}(\varepsilon-1)^{2}}
\end{gathered}
$$

The inspection of the above equations reveals that it has two resonance densities one is the high resonance and another is the low resonance density given by

$$
\begin{aligned}
& n_{H}=\frac{2 n_{c}}{1-\left(\frac{a}{b}\right)} \\
& n_{L}=\frac{2 n_{c}}{1+\left(\frac{a}{b}\right)}
\end{aligned}
$$

It is easily seen that the resonance densities are tuneable depending on the instantaneous values of ' $a$ ' and ' $b$ '. Higher degree of hollowness means resonance density can be close to solid density.

Similar to nano-tubes the radial and angular averaged electric field inside a nano-shell (inner radius ' $a$ ' and outer radius 'b') like "fullerene" is given by

$$
\begin{gathered}
\left\langle E_{\text {in }}\right\rangle=\sqrt{C_{1}^{2}+\frac{2 D_{1}^{2}}{b^{3} a^{3}}} \quad \text { (12) } \\
C_{1}=\frac{-3 E_{0}(2 \varepsilon+1)}{D}, D_{1}=\frac{-3 E_{0} a^{3}(\varepsilon-1)}{D} \\
\mathrm{D}=(\varepsilon+2)(2 \varepsilon+1)-2 \alpha(\varepsilon-1)^{2}
\end{gathered}
$$

$\alpha$ is the degree of hollowness $=\mathrm{a}^{3} / b^{3}$

The inspection of the above equations again reveals that hollow nano-shells too has multiple resonance densities one is the high resonance and another is the low resonance density given by

$$
\begin{aligned}
& n_{H}=\frac{(9+3 \sqrt{1+8 \alpha}) n_{c}}{4(1-\alpha)} \\
& n_{L}=\frac{(9-3 \sqrt{1+8 \alpha}) n_{c}}{4(1-\alpha)}
\end{aligned}
$$




\section{RESonance In Pointed NANO-STRUCTUReS}

The case of nano-elliptical cylinder plasma dielectric kept in laser field with laser polarisation perpendicular to the axis is a little involved. Here the Laplace equation has to be solved in elliptical coordinates; for brevity we just provide the final expression of the inside electric field shown in eq. (17). Resonance condition is obtained from this and it clearly shows that more pointed elliptical cylinder $\mathrm{a}>>\mathrm{b}$ has large resonance density.

$$
E_{\text {in }}=\frac{(1+a / b) E_{0}}{\varepsilon+a / b}
$$

The resonance density is given by

$$
n_{e}=\left(1+\frac{a}{b}\right) n_{c}
$$

It should be mentioned here that nano-cylinder can become like a nano-elliptical cylinder due to polarisation pressure. This will be discussed later.

\section{HYDRODYNAMIC EXPANSION OF NANO-PLASMA}

The laser heated matter is ionized to solid density $\mathrm{n}_{0}$ at the foot of the intense pulse. At lower intensity there may be drastic variation in the maximum initial electron density, but at higher intensities $>10^{15} \mathrm{~W} / \mathrm{cm}^{2}$ the initial electron density $\mathrm{n}_{0}$ is almost constant due to ionization saturation [21].At high intensities due to the onset of hydrodynamic expansion the instantaneous electron density only depends majorly on the plasma expansion speed $\mathrm{c}_{\mathrm{s}}$. The net pressure that causes expansion is due to a sum total of Hydrodynamic, Coulomb and Polarization pressure

$$
P=n k_{B} T_{e}+P_{\text {coulomb }}+P_{\text {Polarisation }}
$$

Hydrodynamic pressure is predominant for large nanoparticles. The plasma expansion speed is given by

$$
\mathrm{c}_{\mathrm{s}}=\left(\frac{\mathrm{Z} \mathrm{k}_{B} \mathrm{Te}}{\mathrm{M}_{i}}\right)^{1 / 2}=10^{4}(T(e V))^{1 / 2}\left(\frac{Z^{*}}{Z}\right)\left(\frac{m}{s}\right)(20)
$$

Here $\mathrm{Z}^{*}$ is average degree of ionization and $\mathrm{Z}$ is the atomic number. The outward expansion of plasma means the radius changes as shown in eq.(21)

$$
a=a_{0}+c_{s} t ; b=a_{0}+c_{s} t
$$

Where ' $a$ ' and ' $b$ ' are time dependent quantities for hollow particles ' $a$ ' is inner and ' $b$ ' is outer radius. In elliptical cylinder ' $a$ ' is major axis along polarization and ' $b$ ' is minor axis perpendicular to laser polarization. $T_{e}$ is shown to depend on laser intensity given by eq.(22) given by Riley et.al [24].

$$
\mathrm{T}(\mathrm{eV})=9.05 \times 10^{-7}\left[\mathrm{I}_{\mathrm{abs}}\left(\mathrm{W} \mathrm{cm}^{-2}\right)\right]^{0.615}
$$

$\mathrm{c}_{\mathrm{s}}$ is assumed to be constant during the hydrodynamic expansion of nano-plasma as shown by R. Issac et.al. [25] They have done experiments where the laser pulse duration was varied from $60-2200 \mathrm{fs}$ at Fluence of $8 \mathrm{~kJ} / \mathrm{cm}^{2}$. They showed the electron temperature was nearly constant and varied from $\sim 1.5 \mathrm{keV}$ to $2 \mathrm{keV}$. In this temperature range the nano-plasma expansion velocity $c_{s}$ varies from 270-320 $\mathrm{nm} / \mathrm{ps}$. It may be noted though variation of pulse duration has little impact on average temperature, but the fluence/ intensity change (By varying the laser energy) cause a drastic change in $T_{e}$ as shown in eq. (22). To find the effect of laser intensity $c_{s}$ can be obtained using eq. (20) and (22)for evaluating the hydrodynamic time scales of resonance of nanostructures of different shapes and geometries. The conservation of the total number of electrons on hydrodynamic expansion, assuming no recombination in laser "on" condition helps in analytically estimating the resonance time scales.

\section{a) Solid Spherical nano/gas clusters}

Conservation of electron number yield

$$
\frac{4}{3} \pi a_{0}{ }^{3} n_{0}=\frac{4}{3} \pi a_{0}{ }^{3} n(t)
$$

From the resonance condition obtained in eq. (5) time scale of resonance $\tau_{\mathrm{R}}$ is given by eq. (24) using eq.(21) and (23)

$$
\tau_{R}=\left(a_{0} / c_{s}\right)\left(\sqrt[3]{\frac{n_{0}}{3 n_{c}}}-1\right)
$$

b) Solid Cylinderical nano/gas clusters

Conservation of electron number for nano-rod

$$
\pi a_{0}{ }^{2} h n_{0}=\pi a^{2} h n(t)
$$

' $h$ ' is height of nano-tube. From the resonance condition obtained in eq. (7) time scale of resonance $\tau_{R}$ is given by eq. (26)using eq.(21) and (25) 


$$
\tau_{R}=\left(a_{0} / c_{s}\right)\left(\sqrt{\frac{n_{0}}{2 n_{c}}}-1\right)
$$

\section{c) Hollow Cylinderical Nano-tube}

Conservation of electron number for nano-tube

$$
\pi\left(b_{0}^{2}-a_{0}^{2}\right) h n_{0}=\pi\left(b^{2}-a^{2}\right) h n(t)
$$

' $h$ ' is height of nano-tube. From the two resonance condition obtained in eq. (10) and (11)the two time scale of resonance $\tau_{\mathrm{H}}$ and $\tau_{\mathrm{L}}$ is given by eq. (28) and (29)using eq.(21) and (27)

$$
\begin{aligned}
& \tau_{R H}=\frac{-(\alpha+\beta)+\sqrt{(\alpha+\beta)^{2}-4 \alpha \beta(1-\gamma)}}{2 \alpha \beta} \\
& \alpha=\frac{2 c_{s}}{a_{0}+b_{0}} ; \beta=\frac{c_{s}}{b_{0}} ; \gamma=\frac{n_{0}}{2 n_{c}}\left(1-\frac{a_{0}}{b_{0}}\right)
\end{aligned}
$$

In case degree of hollowness is high $\tau_{\mathrm{RH}}$ can be approximated

$$
\tau_{R H}=\left(b_{0} / c_{s}\right)\left(\sqrt{\frac{n_{0}}{2 n_{c}}\left(1-\frac{a_{0}^{2}}{b_{0}^{2}}\right)}-1\right)
$$

The general hydrodynamic resonance time scale for low density resonance (without approximation) is given by

$$
\tau_{R L}=\left(\frac{b_{0}}{c_{s}}\right)\left[\left(1+\frac{a_{0}}{b_{0}}\right) \frac{n_{0}}{2 n_{c}}-1\right]
$$

\section{d) Hollow Nano-shell}

Conservation of electron number for nano-shell

$$
\frac{4 \pi}{3}\left(b_{0}{ }^{3}-a_{0}{ }^{3}\right) n_{0}=\frac{4 \pi}{3}\left(b^{3}-a^{3}\right) n(t)
$$

From the two resonances condition obtained in eq. (15) and (16) the two time scale of resonance $\tau_{H}$ and $\tau_{L}$ is given by eq. (32) and (33). This calculated using eq.(15), (16), (21), (31). The general expression is quite complex so to have a simplistic inference the resonance times are calculated in the approximation of nano-shell having a high degree of hollowness.

$$
\begin{gathered}
\tau_{R H}=\left(b_{0} / c_{s}\right)\left(\left[\frac{n_{0}}{3 n_{c}}\left(1-\frac{a_{0}^{3}}{b_{0}{ }^{3}}\right)\right]^{1 / 3}-1\right) \\
\tau_{L H}=\left(b_{0} / c_{s}\right)\left(\left[\frac{2 n_{0}}{3 n_{c}}\left(1-\frac{a_{0}^{3}}{b_{0}{ }^{3}}\right)\right]^{1 / 3}-1\right)
\end{gathered}
$$

\section{e) Nano-elliptical cylinder}

Till now we considered that hydrodynamic pressure is the dominant contributor (see eq.(19)) in nano-plasma expansion dynamics. Now we will show that polarization pressure (eq.(34))is also strong and causes the nano-particle to expand predominantly along the laser polarization. This leads to the structure becoming progressively more pointed. The nanorod becomes nano-elliptical and nano-sphere becomes nanoellipsoid. As the structure shape modifies and becomes pointes the polarization pressure increases further.

$$
\begin{gathered}
P_{p o l}=\frac{1}{2} \operatorname{Re}\left(\overrightarrow{\sigma \cdot \overrightarrow{E_{a v}}}\right) \text { where } \mathrm{E}_{a v}=\frac{1}{2}\left(\overrightarrow{E_{\text {in }}}+\overrightarrow{E_{\text {out }}}\right) \\
\mathrm{P}_{p o l}=\frac{\varepsilon_{0} E_{0}^{2}}{4} \frac{3-\varepsilon}{\varepsilon+1} \cos ^{2} \theta \text { for nano }- \text { rod }
\end{gathered}
$$

' $\sigma$ ' is surface charge density. The polarization pressure of nano-rod is given by eq. (35). It has strong dependence on electric field of laser and has a $\cos \theta$ dependence, which means the pressure is maximum along laser polarization and is zero perpendicular to it. As seen from eq.(18) larger is the value of 'a' (semi major axis) larger is the resonance density. This naturally implies that the resonance density can be achieved for shorter pulse duration if the asymmetric expansion due to polarization pressure is included. The resonance time scale for nano-elliptical cylinder can be obtained as follows. Conservation of electron number for nano-elliptical cylinder assuming expansion along $\mathrm{E}_{0}$

$$
\pi\left(a_{0}^{2}\right) h n_{0}=\pi a_{0}\left(a_{0}+c_{s} t\right) h n(t)
$$

From eq. (18), (21) and (36) the time scale of resonance is

$$
\begin{gathered}
\tau_{R H}=\frac{-3+\sqrt{1+4 \xi}}{2 \kappa} \\
\xi=\frac{n_{0}}{n_{c}} ; \kappa=\frac{c_{s}}{a_{0}}
\end{gathered}
$$

\section{RESUlts AND DiscusSion}

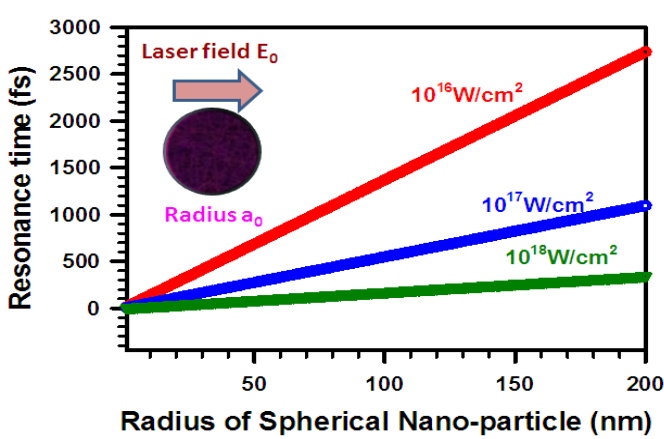

Figure 1. Resonance time in solid spherical cluster as a function of initial size (radius) and intensity of laser irradiation 
Based on the derivation of hydrodynamic time scales of resonance in nano-structures of different geometries as done in the previous section, we now present the typical time scale of resonance in different nano-particles as a function of their geometry and incident laser intensity. The initial electron density close to solid density plasma $\mathrm{n}_{0} / \mathrm{n}_{\mathrm{c}}=40 \quad[20,23]$. Intensity dependence of $\mathrm{c}_{\mathrm{s}}$ is obtained from eq.(20) and (22) is in agreement with predictions of R. Issac et.al[25].

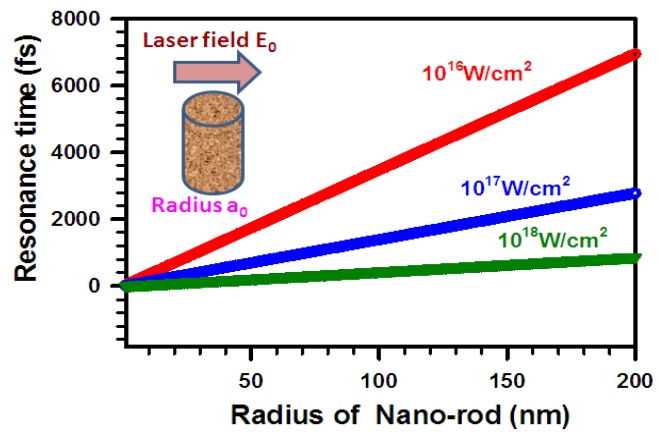

Figure 2. Resonance time in solid nano-rod as a function of initial size (radius) and intensity of laser irradiation

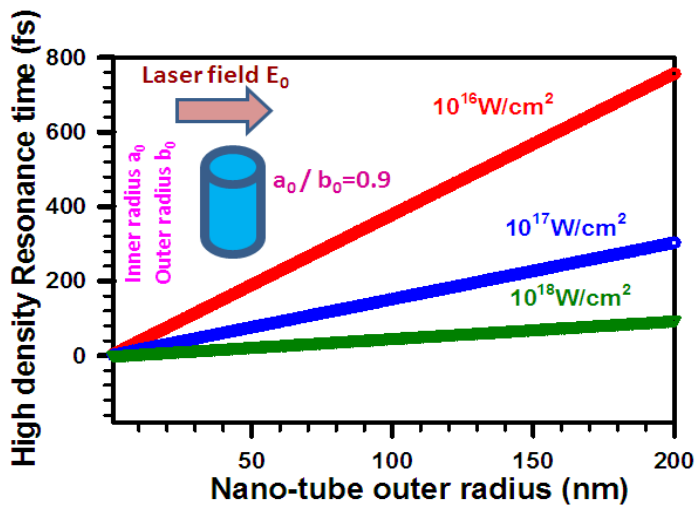

Figure 3. High density resonance time in hollow nano-tube as a function of initial size (outer radius $\mathrm{b}_{0}$ ) and intensity of laser irradiation for $\mathrm{a}_{0} / \mathrm{b}_{0}=0.9$

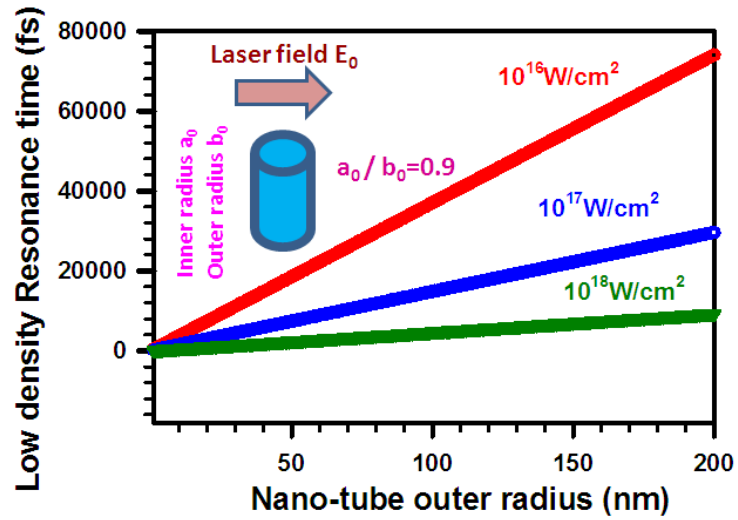

Figure 4. Low density resonance time in hollow nano-tube as a function of initial size (outer radius $b_{0}$ ) and intensity of laser irradiation for $\mathrm{a}_{0} / \mathrm{b}_{0}=0.9$

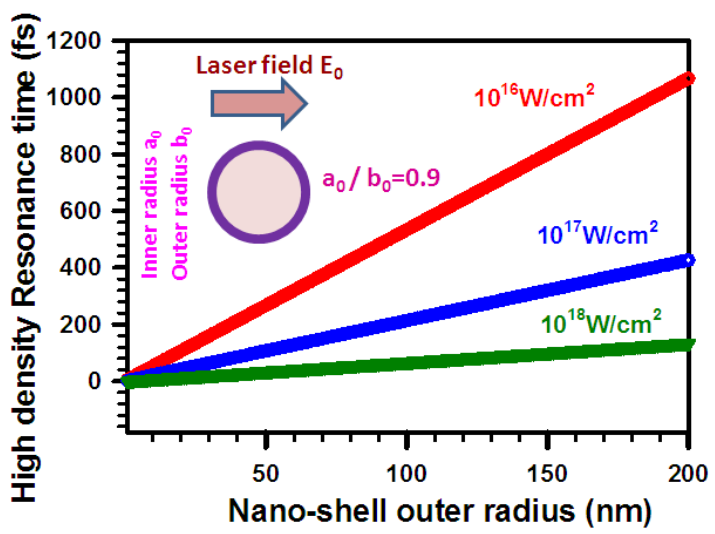

Figure 5. High density resonance time in hollow nano-shell as a function of initial size (outer radius $b_{0}$ ) and intensity of laser irradiation for $a_{0} / b_{0}=0.9$

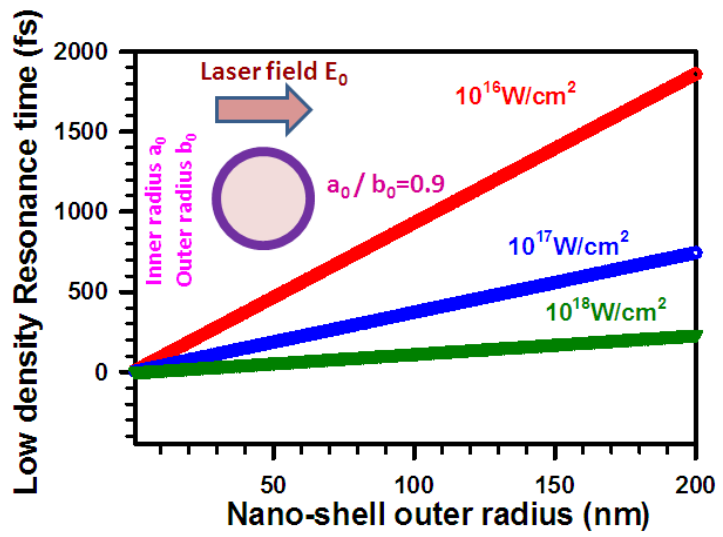

Figure 6. Low density resonance time in hollow nano-shell as a function of initial size (outer radius $b_{0}$ ) and intensity of laser irradiation for $a_{0} / b_{0}=0.9$

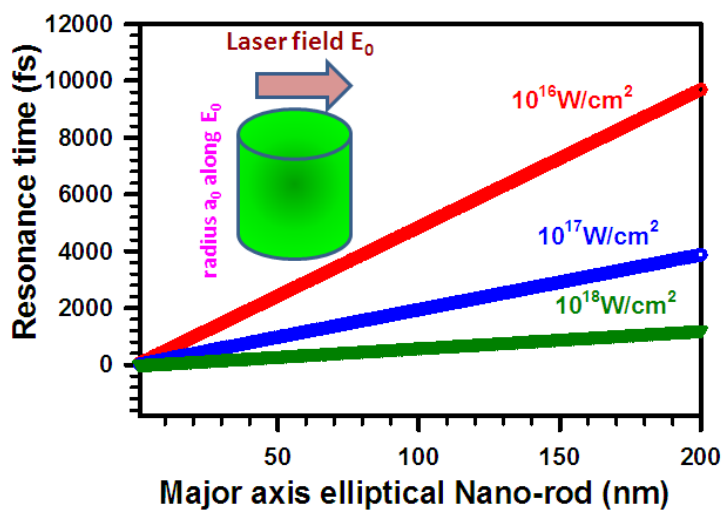

Figure 7. Resonance time in elliptical nanorod as a function of initial size (major axis that is parallel to laser polarisation) and intensity of laser irradiation

After plotting the graphs of hydrodynamic time scales of resonance in nano-structures we now point out and discuss the most noteworthy points. Fig. (1)and (2)shows Resonance time as a function of size (radius) and intensity of laser 
irradiation in solid spherical cluster and nano-rod respectively.They are plotted using eq. (24) and (26) . First and foremost large sized nano-particle reaches resonance later. Therefore longer pulse duration are required to hit resonace at the laser peak. The time scale of resonance in spherical clusters is much larger in cylindrical target of same size. The reason behind is simple that spherical cluster undergoes faster density dilution rate proportional to $1 / \mathrm{r}^{4}$ as compared to cylinder whose density varies as $1 / \mathrm{r}^{3}$. Intensity plays a crucial role in deciding the resonance time and if the available laser pulse is short then a high intensity should be used so that the resonance condition is met at the peak of the laser pulse. Fig.(3) and (5) show that the high density resonance time in hollow nanostructures like nano-tube and nano-shell is drastically small plotted using eq. (29) and (32). This mean if the laser pulse duration is ultrashort hollow nanostructure must be used. In comparison to solid nanoparticle hollow ones have multiple resonance feature. Fig.(4) and (6) shows Low density resonance time in hollow nanotube as a function of initial size (outer radius $b_{0}$ ) and intensity of laser irradiation for $a_{0} / b_{0}=0.9$. They are plotted using eq. (30) and (33). The low density resonance is almost inconsequential for ultrashort pulses as these resonances occur in the ps time scales. However on increasing the laser intensity this resonance may occur during thelaser pulse due to an accelerated expansion. Fig. (7) shows the resonance time in elliptical nanorod as a function of initial size (major axis that is parallel to laser polarisation) and intensity of laser irradiation. The plot is obtained from eq.(37). Here polarisation pressure is assumed to cause expansion at the similar rates as other structures.Only expansion along laser electric field is considered. This one directional expansion causes slower expansion. But if both hydrodynamic and polarisation pressure are included then expansion will occur perpendicular to laser field as well. Though this expansion speed will be less than the expansion along the major axis of elliptical cylinder. Nevertheless a simplistic estimate of the resonance time scale seen from Fig. (7) tell that in elliptical nan-rod a higher intensty is required so that polarisation pressure detrmine the quick arrival of resonance. The analysis given in the paper is a simplistic and comprehensive overview with some major assumptions based on cetrain experimental and theoretical reports available $[9,10,19,21,25]$. Detailed computer simulations will give further insight. Though the derived expression for resonance time scale is quite handy for target design and choice of laser intensity and pulse duration.

\section{CONClusionand Future SCOPE}

In conclusion the following remarks can be made regarding the hydrodynamic time scales of resonance of different nanostructures.
1) Resonance is the key to efficient energy coupling in nano-plasmas and nanostructure geometry decide the density at which resonance will occur and also the field strength.

2) Solid nanostructures like spherical gas clusters $\left(3 n_{c}\right)$ and nanorod $\left(2 n_{c}\right)$ have lower resonance densities and therefore longer time is required for sufficient density dilution on hydrodynamic expansion for achieving the resonance condition.

3) Hollow nanostructures (nano-tubes and shells like CNT and fullerenes)have multiple resonance densities. One is higher resonance density $\left(\mathrm{n}_{\mathrm{H}}\right)$ and another lower $\operatorname{density}\left(\mathrm{n}_{\mathrm{L}}\right)$.Higher resonnace density is achieved forshorter pulse duration and lower resonance density is achievd in longer time. For ultrashort pulses targets of higher degree of hollowness are ideal, since hydrodynamic expansion for density dilution is not a strong pre-requiste.

4) Polarisation pressure causes assymetric expansion and leads to an accelerated expansion predominantly along the laser polarisation. The case of assymetric expansion is shown for nano-cylinderthoughfurther studies are needed for dynamics of nano-particle evolution by including Hydrodynamic, Polarization and Coulomb pressure simultaneously.

5) Laser fluence / Intensity also play a dominant role in determing the hydrodynamic time scales fo resonance due to faster expansion at higher intensities. Higher intensity causes resonance to be achieved earlier in time and therefore stretching of pulse to meet resonance at laser peak is precluded. Hollow and pointed nanostructures resonance can be excited at lower intensity since they have already high resonance density and therfore high intensity requirement for resonance excitation is not too strong.

Generally laser gas cluster [14,21] and nano-rod [16,23] interaction with intense pulses has been studied experimentally and analytically in various Laboratories but hollow nanostructures like nano-tube, nano-shells and pointed nanostructures studies are rather rare. Using them can lead to significant advancement in overcoming the challenge of inefficient coupling of high intensity light in solid density matter. This work and the derived expressions for hydrodynamic time scales of resonance will serve as a guide for choosing laser and target geometrical parameters for efficient laser energy coupling in nano-plasmas for efficient X-rays, electron and ion generation. Also the analytical solutions of fields and resonance densities are useful for plasma and nano-science community [26, 27].

\section{ACKNOWLEDGMENT}

I thank my colleagues at Laser Plasma Section, R.R.C.A.T., Indore for encouraging me in compilation of this work.

\section{REFERENCES}


[1] D. F. Price, R. M. More, R. S. Walling, G. Guethlein, R. L. Shepherd, R. E. Stewart, and W. E. White. "Absorption of ultra-short laser pulses by solid targets heated rapidly to temperatures 1-1000 eV."Physical review letters75, 252(1995).

[2] T. Nishikawa, H. Nakano, K. Oguri, N. Uesugi, K. Nishio, and H. Masuda, "Nano-hole-array size dependence of soft x-ray generation enhancement from femtosecond-laser-produced plasma", J. Appl. Phys.96, 7537 (2004).

[3] T. Ditmire, J. W. G. Tisch, E. Springate, M. B. Mason, N. Hay, R. A. Smith, J. Marangos, and M. H. R. Hutchinson, "High-energy ions produced in explosions of superheated atomic clusters", Nature 386, 54 (1997)

[4] Y. Ji, G. Jiang, W.Wu, C. Wang, Y. Gu, and Y. Tang, "Efficient generation and transportation of energetic electrons in a carbon nanotube array target", Appl. Phys. Lett.96, 041504 (2010).

[5] M. Dunne, "A high-power laser fusion facility for Europe." Nature Physics 2, 2-5 (2006).

[6] S.A. Pikuz, O. V. Chefonov, S. V. Gasilov, P. S. Komarov, A. V. Ovchinnikov, I. Yu Skobelev, S. Yu Ashitkov, M. V. Agranat, A. Zigler, and A. Ya Faenov. "Micro-radiography with laser plasma Xray source operating in air atmosphere. "Laser and Particle Beams: pulse power \& high energy densities28, 393-397 (2010).

[7] J. C. Kieffer, A. Krol, Z. Jiang, C. C. Chamberlain, E. Scalzetti, and Z. Ichalalene, "Future of laser-based X-ray sources for medical imaging."Appl. Phys. B: Lasers Opt.74, S75 (2002).

[8] L. Karsch, E. Beyreuther, W. Enghardt, M. Gotz, U. Masood, U. Schramm, K. Zeil, and J. Pawelke, "Towards ion beam therapy based on laser plasma accelerators”. Acta Oncologica, 56(11), pp.13591366, (2017).

[9] H. M. Milchberg, and R. R. Freeman. "Light absorption in ultra-short scale length plasmas." JOSA B6, 1351-1355 (1989).

[10] E. Parra, I. Alexeev, J. Fan, K. Y. Kim, S. J. McNaught, and H. M. Milchberg,"Hydrodynamic time scales for intense laser-heated clusters." J. Opt. Soc. Am. B, 20, 118 ( 2003).

[11] P. Mulser, and M. Kanapathipillai. "Collisionless absorption in clusters out of linear resonance."Physical Review A71, 063201 (2005).

[12] U. Saalmann, and J.M. Rost. "Ionization of clusters in intense laser pulses through collective electron dynamics."Physical Review Letters91, 223401 (2003).

[13] T. Nishikawa, S. Suzuki, Y. Watanabe, O. Zhou, H. Nakano, "Efficient water-window $x$-ray pulse generation from femtosecond-laserproduced plasma by using a carbon nano-tube target ", Appl. Phys. B78, 885 (2004).

[14] F. Dorchies, F. Blasco, C. Bonté, T. Caillaud, C. Fourment, and O. Peyrusse, "Observation of subpicosecond x-ray emission from lasercluster interaction",Phys. Rev. Lett.100, 205002 (2008).

[15] M. M. Murnane, H. C. Kapteyn, S. P. Gordon, J. Bokor, and E. N. Glytsis, "Efficient coupling of high-intensity subpicosecond laser pulses into solids", Appl. Phys. Lett. 62, 1068, (1993).

[16] G. Kulcsar, D. AlMawlawi, F. W. Bundnik, P. R. Herman, M. Moskovits, L. Zhao, and R. S. Marjoribanks, "Intense picosecond xray pulses from laser plasmas by use of nano-structured "Velvet" targets",Phys. Rev. Lett.84, 5149 (2000).

[17] J. Yanling, G. Jiang, W. Wu, C. Wang, Y. Gu, and Y. Tang. "Efficient generation and transportation of energetic electrons in a carbon nanotube array target." Applied Physics Letters96, 041504-041504 (2010).

[18] P. P. Rajeev, P. Ayyub, S. Bagchi, and G. R. Kumar, "Nanostructures, local fields, and enhanced absorption in intense lightmatter interaction ", Optics Letters29, 22 (2004).

[19] T. Ditmire, T. Donnelly, A. M. Rubenchik, R. W. Falcone, and M. D. Perry, "Interaction of intense laser pulses with atomic clusters, "Phys. Rev. A. 53, 3379 (1996)
[20] U. Chakravarty, P. A. Naik, and P. D. Gupta. "Electric field enhancement at multiple densities in laser-irradiated nanotube plasma."Pramana79, 443-456 (2012).

[21] J. Zweiback, T. Ditmire, and M. D. Perry, "Femtosecond timeresolved studies of the dynamics of noble-gas cluster explosions", Phys. Rev. A59, R3166 (1999).

[22] M. Kumar, and V. K. Tripathi. "Nonlinear absorption and harmonic generation of laser in a gas with anharmonic clusters. "Physics of Plasmas20, 023302 (2013).

[23] U. Chakravarty, P.A. Naik, B.S. Rao, V. Arora, H. Singhal, G.M. Bhalerao, A.K. Sinha, P. Tiwari, and P.D. Gupta,"Enhanced soft X-ray emission from carbon nanofibers irradiated with ultra-short laser pulses."Appl. Phys. B103, 571 (2011).

[24] D. Riley, L. A. Gizzi, A. J. Mackinnon, S. M. Viana, and O. Willi. "Absorption of high-contrast 12-ps uv laser pulses by solid targets. "Physical Review E48, 4855 (1993).

[25] R. C. Issac, G. Vieux, B. Ersfeld, E. Brunetti, S. P. Jamison, J. Gallacher, D. Clark,and D. A. Jaroszynski, "Ultra hard x rays from krypton clusters heated by intense laser fields."Phys. Plasmas 11, 3491 (2004).

[26] U. Sharma, S.S. Chauhan, A.K. Sanyasi, K.K. Chaudhary, J.Sharma, andJ. Ghosh, , "Development of Experimental Setup for Plasma Facilities at SVITS" IJSRPAS, 3, 22-26 (2018).

[27] K.E. Kuppan, K. Sakthiumurugesan"A study on the properties Synthesis, Growth, spectral, structural, optical thermal studies of a new organic crystal \& NLO applications of $\mathrm{N}$-(tert-butyl)-4(nitrophenyl imidazo[1,2-a]pyrazin-3-amine", IJSRPAS, 6, 55-63 (2018).

\section{AUTHORS PROFILE}

Dr. UdayChakravarty did his M.Sc. from J.N.U. New Delhi in 2003 and Ph.D. from H.B.N.I in Physical Sciences in 2014. He is working at R.R.C.A.T. Indore (India) as Scientific Officer F in Laser Plasma Section. $\mathrm{He}$ is also appointed as Assistant Professor in H.B.N.I. His research interest includes laser matter interaction studies. Laser microstructure and nano-structure experiments and analytical modelling. He has published various research papers in reputed international journals. He has 10 years of teaching experience in BARC Training School at RRCAT. He is recipient of BUTI Young Scientist award given by the Buti foundation, Plasma Science Society of India.

Deepa Chaturvedi has done M.Sc. and M.Tech from Devi Ahilya University, Indore, Madhya Pradesh and was the university topper. She is pursuing her Ph.D. in Physics from I.I.T. Delhi. Her expertise is on analytical studies of Nano-optics and Silicon nano-waveguides. She has published many papers in reputed international journals

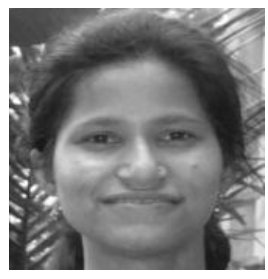
and conferences. 